\title{
FREQUENCY RESPONSE OF A THERMOCOUPLE WIRE : EFFECTS OF AXIAL CONDUCTION
}

\author{
Progress Report \\ April 1990 - September 1990 \\ L. J. Forney \\ School of Chemical Engineering \\ Georgia Institute of Technology \\ Atlanta, Georgia 30332 \\ and \\ G. C. Fralick \\ Research Sensor Technology Branch \\ NASA - Lewis Research Center \\ Cleveland, Ohio 44135
}

p. 40

Sponsored by

ARNOLD ENGINEERING DEVELOPMENT CENTER

Arnold Air Force Base, Tennessee 37389

under

NASA Cooperative Agreement NCC 3-135

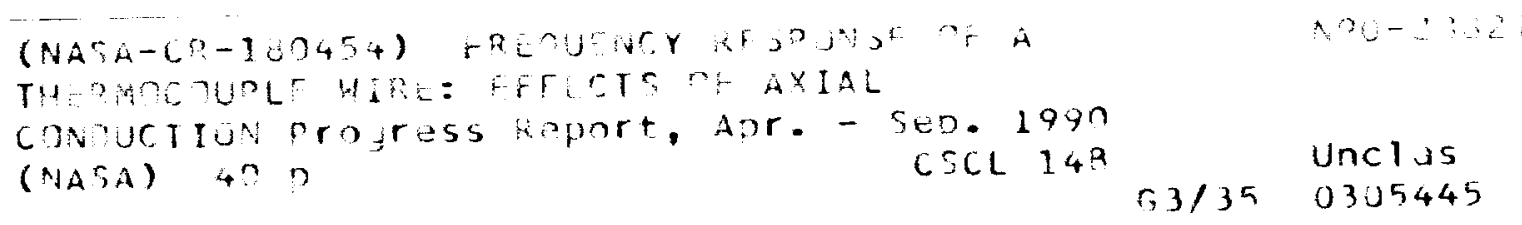

Georgia Institute of Technology

September, 1990 
Frequency Response of a Thermocouple Wire :

Effects of Axial Conduction

\author{
L. J. Forney \\ School of Chemical Engineering \\ Georgia Institute of Technology \\ Atlanta, Georgia 30332 \\ G. C. Fralick \\ Research Sensor Technology Branch \\ NASA - Lewis Research Center \\ Cleveland, Ohio 44135
}

Georgia Institute of Technology

September, 1990 


\begin{abstract}
Theoretical expressions are derived for the steady-state frequency response of a thermocouple wire. In particular, the effects of axial heat conduction are demonstrated for both a uniform thermocouple wire and a non-uniform wire with unequal material properties and wire diameters across the junction. For the case of a uniform wire, the amplitude ratio and phase angle compare favorably with the series solution of Scadron and Warshawsky (1952) except near the ends of the wire. For the case of a non-uniform wire, the amplitude ratio at low frequency $\omega \rightarrow 0$ agrees with the results of Scadron and Warshawsky for a steady-state temperature distribution. Moreover, the frequency response for a non-uniform wire in the limit of infinite length $l \rightarrow \infty$ is shown to reduce to a simple expression that is analogous to the classic first order solution for a thermocouple wire with uniform properties.
\end{abstract}





\section{INTRODUCTION}

The evaluation of jet engine performance and fundamental studies of combustion phenomena depend on the measurement of turbulent fluctuating temperatures of the gas within the engine (Dils and Follansbee, 1976). Historically, these temperatures have been measured with thermocouples. The design of a thermocouple represents a compromise between accuracy, ruggedness and rapidity of response.

For example, the measurement of fluctuating temperatures in the high speed exhaust of a gas turbine engine combustor is required to characterize the local gas density gradients or convective heat transfer (Fralick, 1985). Although thermocouples are suitable for the measurement of high frequency temperature fluctuations $(<1 \mathrm{KHz})$ in a flowing gas or liquid, the measured signal must be compensated since the frequency of the time dependent fluid temperature is normally much higher than the corner frequency of the thermocouple probe (Scadron and Warshawsky, 1952). Moreover, the amplitude and phase angle of the thermocouple response may be attenuated by axial heat conduction for rugged thermocouples of finite length (Elmore, et al.; 1983, 1986).

In the present study, the theoretical steady-state frequency response of a thermocouple wire has been calculated to include the effects of axial heat conduction. Solutions are derived for both a uniform wire and a non-uniform wire with unequal properties across the junction (e.g., different wire diameter, 
thermoconductivity, etc.). Solutions are presented in the form of the amplitude ratio and phase angle for both the uniform and non-uniform thermocouple wires. In both configurations, the results are presented for thermocouple wires of either finite length where the effects of axial heat conduction are demonstrated or wires of infinite length for which the neglect of axial conduction is justified. For the case of the uniform wire, the theoretical results are compared with the series solutions of Scadron and Warshawsky (1952). Moreover, for non-uniform wires the results are compared in the limit of low frequency with the steady-state temperature solution of Scadron and Warshawsky.

\section{THEORY}

The steady-state frequency response of a thermocouple wire will be developed in the following sections with the following assumptions: (a) the amplitude of the fluctuating fluid temperature is small relative to the mean absolute temperature (b) the thermocouple dimensions are small relative to the size of the turbulent eddies or enclosure dimensions (c) radial temperature gradients in a wire cross section can be neglected and (d) radiative heat transfer can be neglected relative to conduction and convection.

\section{Uniform Wire}

In this section the geometry of Fig. 1 is considered where the material properties of thermal conductivity $k$, specific heat $c$ and wire density $\rho$ are 
assumed to be equal on both sides of the thermocouple junction. If the probe is subjected to a flowing fluid, whose temperature fluctuates about its mean, the expression for the local conservation of energy in the thermocouple wire becomes (Scadron and Warshawsky, 1952)

$$
\frac{\partial T_{\mathrm{w}}}{\partial t}=\alpha \frac{\partial^{2} T_{\mathrm{w}}}{\partial x^{2}}+\frac{4 h}{\rho c D}\left(T_{\mathrm{s}}-T_{\mathrm{w}}\right)
$$

where $\alpha=k / \rho c$ is the thermal diffusivity of the wire, $\mathrm{T}_{8}$ is the ambient fluid temperature, $h$ is the convective heat transfer coefficient, $D$ is the wire diameter and $T_{w}$ is the local wire temperature measured along the axis at a distance $x$ from the centerline of Fig. 1.

If the wire and fluid temperatures are measured relative to the support and mean fluid temperature $T_{0}$, the variation in the ambient fluid temperature can be written in the complex form

$$
T_{f}(t)=T_{0}+T_{f} e^{i \omega t}
$$

where $\omega$ is the angular frequency of the ambient fluid. Since Eq. (1) is linear, we now seek a solution for the local wire temperature of the form (Hildebrand, 1976)

$$
T_{w}=T_{0}+\vec{T}_{\omega}(x) e^{i \omega t}
$$

Referencing all temperatures with respect to the steady-state temperature $T_{0}$ and 
normalizing with respect to the amplitude of the fluctuating ambient fluid temperature $T_{f}$, one defines a local normalized steady-state frequency response $\vec{T}(x)$ for the thermocouple wire of the form

$$
\frac{T_{w}-T_{0}}{T_{f}}=\frac{\vec{T}_{\omega}(x) e^{i \omega t}}{T_{f}}=\vec{T}(x) e^{i \omega t}
$$

Substituting Eqs. (2) and (3) into Eq. (1), one obtains an ordinary differential equation of the form

$$
i \omega T=\alpha \frac{d^{2} T}{d x^{2}}+\omega_{\wedge}(1-T)
$$

where $T=\vec{T}(x)$ is the frequency response and the vector notation will be dropped for simplicity. Thus for the geometry of fig. 1, one seeks a solution to the nonhomogeneous linear second order differential equation for the dependent variable $\mathrm{T}$ of the form

$$
\gamma T^{\prime \prime}-G(\omega) T=-1
$$

subject to the boundary conditions

$$
T(l)=T(-l)=0
$$

Here, the parameters in Eqs. (5) and (6) are defined as 


$$
\omega_{n}=\frac{4 h}{\rho c D}, \quad \gamma=\frac{\alpha}{\omega_{n}}, \quad G(\omega)=1+i\left(\frac{\omega}{\omega_{n}}\right)
$$

where $\omega_{n}$ is the natural frequency of the uniform wire of fig. 1 .

The general solution to Eq.(6) can be written in the form (Hildebrand, 1976)

$$
T=A \sinh q x+B \cosh q x+\frac{1}{G(\omega)}
$$

where the constants A and B are complex, $1 / G(\omega)$ represents the particular solution and the parameter

$$
q=\sqrt{\frac{G(\omega)}{\gamma}}
$$

Substituting $x= \pm l$ in Eq. (9), the boundary condition Eq. (7) yields values for the constants

$$
A=0, \quad B=\frac{-1}{G(\omega) \cosh q l}
$$

Hence, one obtains a steady-state frequency response for the simple uniform wire of Fig. 1 in the form (Forney, 1988)

$$
T=\frac{1}{G(\omega)}\left(1-\frac{\cosh q x}{\cosh q l}\right) .
$$


The steady-state frequency response at the junction $x=0$ of fig. 1 now becomes

$$
T(0)=\frac{1}{G(\omega)}(1-\operatorname{sech} q l)
$$

The term $1-\operatorname{sech}(q l)$ in Eq. (12) corresponds to the steady-state temperature distribution for a constant ambient fluid temperature $T_{f}>T_{0}$ derived by Scadron and Warshawsky (1952). It is also interesting to note that the first order response can be derived from Eq.(12) in the limit of large wire length $l$ or

$$
\lim _{i \rightarrow \infty} T(0)=\frac{1}{G(\omega)}
$$

where Eq. (13) represents the frequency response of a uniform thermocouple wire with no axial heat conduction.

The steady-state frequency at the thermocouple junction $x=0$ is normally characterized graphically in the form

$$
T(0)=|T(0)| e^{i \oplus}
$$

where $|T(0)|$ is the amplitude ratio and $\Phi$ is the phase angle. In the latter case, the phase angle in degrees is

$$
\Phi=57.3 \tan ^{-1}\left[\frac{\operatorname{Im} T(0)}{\operatorname{Re} T(0)}\right]
$$


where $\operatorname{Im}[T(0)]$ and $\operatorname{Re}[T(0)]$ are the imaginary and real parts of $T(0)$, respectively.

\section{Previous Work}

Scadron and Warshawsky (1952) in their pioneering work considered the frequency response of a uniform thermocouple wire as shown in fig. 1 . Introducing transformations for the dependent variable $T_{w}$ in Eq. (1) as suggested by Carslaw (1945), Scadron and Warshawsky obtained expressions for the steadystate frequency response in the form of the amplitude ratio

$$
|T(0)|=\frac{\sqrt{S_{1}^{2}+\left(\frac{\omega}{\omega_{n}}\right)^{2} S_{2}^{2}}}{\sqrt{1+\left(\frac{\omega}{\omega_{n}}\right)^{2}}}
$$

and phase angle

$$
\Phi=\tan ^{-1}\left(\frac{\omega S_{2}}{\omega_{n} S_{1}}\right)-\tan ^{-1}\left(\frac{\omega}{\omega_{n}}\right)
$$

Here, the infinite series $S_{1}$ and $S_{2}$ that appear in Eqs. (16) and (17) are 


$$
S_{1}=1-\sum_{j=1} c_{j}\left[\frac{d_{j}\left(d_{j}-1\right)}{d_{j}^{2}+\left(\frac{\omega}{\omega_{n}}\right)^{2}}\right]
$$

and

$$
S_{2}=\sum_{j=1}^{\infty} c_{j}\left[\frac{d_{j}-1}{d_{j}{ }^{2}+\left(\frac{\omega}{\omega_{n}}\right)^{2}}\right]
$$

where the constants that appear in the terms of $S_{1}$ and $S_{2}$ are

$$
c_{j}=\frac{2}{j \pi}[1-\cos (j \pi)] \sin \left(\frac{j \pi(l-x)}{2 l}\right)
$$

and

$$
d_{j}=1+\frac{(j \pi)^{2} \gamma}{4 l^{2}}
$$

Properties of these solutions derived by Scadron and Warshawsky are discussed in Sec. W.

\section{Non-Uniform Wire}

If the wire diameters or the physical properties such as thermoconductivity are different across the thermocouple junction as shown in fig.2, Eq.(6) must be 
solved in the separate regions 1 and 2 on both sides of the junction. This solution is subject to the boundary conditions of continuous temperature and equal rates of conductive heat transfer at the junction $x=0$ or

$$
\begin{gathered}
T_{1}(-l)=T_{2}(l)=0 \\
T_{1}(0)=T_{2}(0) \\
k_{1} D_{1}^{2} \frac{d T_{1}(0)}{d x}=k_{2} D_{2}^{2} \frac{d T_{2}(0)}{d x} .
\end{gathered}
$$

In region 1, the general solution to Eq. (6) for the frequency response can be written in the form

$$
T_{1}(x)=A_{1} \sinh q_{1}(l+x)+B_{1} \cosh q_{1}(l+x)+\frac{1}{G_{1}(\omega)}
$$

Since $T_{1}(-l)=0$ from boundary condition Eq. (20a), one finds for the constant $B_{1}$

$$
0=B_{1}+\frac{1}{G_{1}(\omega)}
$$

Substituting for $B_{1}$ into Eq. (21), one obtains 


$$
T_{1}(x)=A_{1} \sinh q_{1}(l+x)+\frac{1}{G_{1}(\omega)}\left[1-\cosh q_{1}(l+x)\right]
$$

where the parameters in Eq. (22) reflect the material properties and wire diameter of region 1 or

$$
\omega_{1}=\frac{4 h_{1}}{\rho_{1} c_{1} D_{1}}, \quad \gamma_{1}=\frac{\alpha_{1}}{\omega_{1}}, \quad G_{1}(\omega)=1+i\left(\frac{\omega}{\omega_{1}}\right), \quad q_{1}=\sqrt{\frac{G_{1}(\omega)}{\gamma_{1}}} .
$$

Similarly in region 2, the frequency response can be shown to be of the form

$$
T_{2}(x)=A_{2} \sinh q_{2}(l-x)+\frac{1}{G_{2}(\omega)}\left[1-\cosh q_{2}(l-x)\right]
$$

where the constants $A_{1}$ and $A_{2}$ in Eqs. (22) and (23) above must be determined by the simultaneous solution to the boundary conditions Eqs. (20b) and (20c).

Substituting Eqs. (22) and (23) into boundary condition (20b), one obtains a linear equation for the constants of integration $A_{1}, A_{2}$ or

$$
A_{1} \sinh q_{1} l-A_{2} \sinh q_{2} l=R_{1} \text {. }
$$

Similarly, substituting Eqs. (22) and (23) into boundary condition (20c), one obtains a second linear equation for $A_{1}, A_{2}$ or

$$
A_{1} Q \cosh q_{1} l+A_{2} \cosh q_{2} l=R_{2} \text {. }
$$


Here, the complex constant $Q$ is defined as

$$
Q=\frac{k_{1} D_{1}^{2} q_{1}}{k_{2} D_{2}^{2} q_{2}}
$$

and the complex constants $R_{1}, R_{2}$ are equal to

$$
R_{1}=\left(\frac{1}{G_{2}}-\frac{1}{G_{1}}\right)+\frac{1}{G_{1}} \cosh q_{1} l-\frac{1}{G_{2}} \cosh q_{2} l
$$

and

$$
R_{2}=\frac{Q}{G_{1}} \sinh q_{1} l+\frac{1}{G_{2}} \sinh q_{2} l
$$

Solving Eqs. (24) and (25) for $A_{1}$ and $A_{2}$ one obtains the determinate system

$$
A_{1}=\frac{\left|\begin{array}{cc}
R_{1} & -\sinh q_{2} l \\
R_{2} & \cosh q_{2} l
\end{array}\right|}{D E T}
$$

and

$$
A_{2}=\frac{\left|\begin{array}{ll}
\sinh q_{1} l & R_{1} \\
Q \cosh q_{1} l & R_{2}
\end{array}\right|}{D E T}
$$


where the determinate in the denominator is equal to

$$
D E T=\sinh q_{1} l \cosh q_{2} l+Q \cosh q_{1} l \sinh q_{2} l
$$

Thus, the constants $A_{1}$ and $A_{2}$ in Eqs. (22) and (23) become

$$
A_{1}=\frac{\frac{\sinh q_{1} l}{G_{1}}\left(\tanh \frac{q_{1} l}{2}+Q \tanh q_{2} l\right)+\frac{\sinh q_{2} l}{G_{2}}\left(-\tanh \frac{q_{2} l}{2}+\tanh q_{2} l\right)}{\sinh q_{1} l+Q \cosh q_{1} l \tanh q_{2} l}
$$

and

$$
A_{2}=\frac{\frac{\sinh q_{1} l}{G_{1}}\left(\tanh q_{1} l-\tanh \frac{q_{1} l}{2}\right)+\frac{\sinh q_{2} l}{G_{2}}\left(\tanh \frac{q_{2} l}{2}+Q^{\prime} \tanh q_{1} l\right)}{\sinh q_{2} l+Q^{\prime} \cosh q_{2} l \tanh q_{1} l}
$$

where the complex constant in $A_{2}$ is

$$
Q^{\prime}=1 / Q
$$

Finally, the steady-state frequency response at the thermocouple junction $x$ $=0$ can be determined by substituting the value for $\mathrm{A}_{1}$ above or Eq. (32) into the general solution for $\mathrm{T}_{1}(0)$ given by Eq. (22). One obtains after simplification

$$
T_{1}(0)=\frac{\frac{Q \sinh q_{1} l}{G_{1}}\left(1-\operatorname{coth} q_{1} l \tanh \frac{q_{1} l}{2}\right)+\frac{\sinh q_{2} l}{G_{2}}\left(1-\operatorname{coth} q_{2} l \tanh \frac{q_{2} l}{2}\right)}{Q \operatorname{coth} q_{1} l+\operatorname{coth} q_{2} l}
$$


where it can be shown that $T_{1}(0)=T_{2}(0)$ at the thermocouple junction.

It is interesting to note that one can determine a frequency response for a non-uniform wire of infinite length from Eq. (34) in a manner similar to the derivation of Eq. (13). Taking the limit of infinite wire length in $T_{1}(0)$ above, one obtains the simple expression

$$
\lim _{i \rightarrow-} T_{1}(0)=\frac{\frac{Q}{G_{1}}+\frac{1}{G_{2}}}{Q+1}
$$

Equation (35) represents the frequency response for a non-uniform wire which is analogous to the well known first order response given by Eq. (13) for a uniform wire of infinite length.

\section{RESULTS}

The amplitude ratio and phase angle of the thermocouple frequency response were plotted graphically for the case of a uniform wire with the geometry shown in fig. 1 . In this case, average properties of a type $B$ or

$P t / 6 \% R h-P t / 30 \% R h$ were used since the material properties were nearly equal across the thermocouple junction. The wire dimensions, properties and gas conditions are listed in table 1(Touloukian et al., 1970).

The amplitude ratio and phase angle were also plotted for a composite or 
non-uniform thermocouple wire with the geometry shown in fig. 2 . In this case, different wire diameters and material properties were used including a type B thermocouple described in Table 1 in addition to a type $\mathrm{T}$ or copper - constantan described in table 2 (Touloukian et al., 1970).

The form of the convective heat transfer coefficient $h$ that appears in the computation of the natural frequency $\omega_{n}$ defined in Eq. (8) was determined from the expression (Scadron and Warshawsky 1952)

$$
N u=.431 \mathrm{Re}^{1 / 2}
$$

where $N u\left(=h D / k_{f}\right)$ is the Nusselt number, $\mathrm{k}_{\mathrm{f}}$ is the thermal conductivity of the ambient fluid and $\operatorname{Re}\left(=v D / v_{f}\right)$ is the Reynolds number of the thermocouple wire. Here, $\mathbf{v}$ and $v$, are the fluid velocity and kinematic viscosity, respectively. It should be noted that the convective heat transfer coefficient $h \alpha D^{1 / 2}$ and the natural frequency of a thermocouple wire for given material properties $\omega_{n} \alpha D^{-3 / 2}$

\section{Uniform Wire}

The amplitude ratio $|T(0)|$ at the wire junction for the steady-state frequency response Eq. (12) is computed in fig. 3. This assumes a type B thermocouple wire with the dimensions listed in table 1 . In this case, the average material properties listed in table 1 were used since the theory assumes that the 
thermocouple is uniform across the junction. In fig. 3 the amplitude ratio $|T(0)|$ $\rightarrow .54$ for low frequencies $\omega \rightarrow 0$. This lower limit corresponds to the attenuation expected for a thermocouple wire exposed to a steady ambient gas temperature $T_{f}$ $>\mathrm{T}_{0}$ as derived by Scadron and Warshawsky (1952).

The amplitude ratio Eq. (16) predicted by Scadron and Warshawsky (1952) is also graphed in fig. 3. It was found that the series Eqs. (18a) and (18b) converged slowly such that the final result was in error by $\sim 11 \%$ at low frequencies after retaining the first 10 terms. The first 50 terms of the series expressions for $S_{1}$ and $\mathrm{S}_{2}$ reduced the error to $2.3 \%$ while retaining 100 terms as was done in fig. 3 limited the error to $1 \%$. The final result is identical, however, to the amplitude ratio predicted by Eq. (12) and derived in the present study.

The amplitude ratio for a type $B$ thermocouple wire of infinite length from the first order response Eq. (13) is also indicated in fig. 3. As expected, the first order response or Eq. (13) asymptotically approaches the amplitude ratio predicted for a wire of finite length in the limit of large frequencies $\omega / \omega_{n} \gg 1$ where the time scale of the ambient temperature fluctuation is too small for axial heat conduction to occur.

The phase angle $\Phi$ for all three theories is computed in fig. 4. The phase angle varies over the range $0 \geq \Phi \geq-\pi / 2$ and approaches the lower limit of $-\pi / 2$ for large frequencies $w / w_{n} \gg 1$. It is apparent that the effect of axial heat conduction is to reduce the magnitude of the phase angle. 
The spacial variation of the amplitude ratio $|T(x)|$ derived from Eq. (11) is graphed in fig. 5. The present theory approaches the correct limit of $|T( \pm l)|=0$. However, the theory of Scadron and Warshawsky oscillates near the end of the wire $x \rightarrow \pm l$. In the latter case the amplitude ratio equals the value $1 / \sqrt{1+\left(\omega / \omega_{n}\right)^{2}}$ for $x= \pm l$ since $S_{1}=1$ and $S_{2}=0$ in Eqs. (18a) and (18b), respectively. An incorrect value for the amplitude ratio near the ends of the wire would not ordinarily present a problem unless one wished to adapt the theory to the common configuration of a supported thermocouple wire with heat transfer down the support legs (Fralich and Forney, 1990).

\section{Non-Uniform Wire}

The amplitude ratio $\left|T_{1}(0)\right|$ from the steady-state frequency response Eq. (34) is graphed in fig. 6. . This plot assumes a type $T$ thermocouple wire with the dimensions listed in table 2 . An important parameter that affects the relative magnitude of the terms in Eq. (34) and thus the relative importance of the thermocouple wire segments in regions 1 or 2 in the schematic of fig. 2 is the complex dimensionless ratio $Q=k_{1} D_{1}^{2} q_{1} / k_{2} D_{2}^{2} q_{2}$. We note here that $Q$ assumes different values in the limit of either low or high frequencies. Given that the Nusselt number is related to the wire Reynolds number by Eq. (36), one obtains for low frequencies

$$
Q(\omega=0)=Q_{0}=\left(\frac{k_{1}}{k_{2}}\right)^{.5}\left(\frac{D_{1}}{D_{2}}\right)^{1.25}
$$


while for high frequencies

$$
Q(\omega=\infty)=Q=\left(\frac{k_{1}}{k_{2}}\right)^{s}\left(\frac{D_{1}}{D_{2}}\right)^{2} .
$$

The amplitude ratio $\left|T_{1}(0)\right| \rightarrow .228$ for low frequencies $\omega \rightarrow 0$ in fig. 6 . The lower limit corresponds to the attenuation predicted by Scadron and Warshawsky (1952) for a non-uniform thermocouple wire as shown in fig. 2 that is exposed to a steady-state ambient gas temperature $T_{f}>T_{0}$. The real expression for the temperature distribution developed by Scadron and Warshawsky for this case is

$$
\frac{T_{w}(0)-T_{0}}{T_{f}}=1-\psi
$$

where

$$
\psi=\operatorname{sech} \frac{l \gamma^{+}}{2} \cosh \frac{l \gamma^{-}}{2}\left[\frac{1-m_{12} \tanh \frac{l \gamma^{-}}{2} \operatorname{coth} \frac{l \gamma^{+}}{2}}{1-m_{12} \sinh l \gamma^{-} \operatorname{csch} l \gamma^{+}}\right] .
$$

Here, the parameters $\gamma^{+}$and $\gamma^{-}$are defined as

$$
\gamma^{+}=\frac{1}{\sqrt{\gamma_{1}}}+\frac{1}{\sqrt{\gamma_{2}}}, \quad \gamma^{-}=\frac{1}{\sqrt{\gamma_{1}}}-\frac{1}{\sqrt{\gamma_{2}}}
$$

and 


$$
m_{12}=\frac{Q_{0}-1}{Q_{0}+1}
$$

The amplitude ratio $\left|T_{1}(0)\right|$ from Eq. (35) for a type $T$ thermocouple wire with the same wire dimensions $D_{2} / D_{1}=2$ as the solid curve in fig. 6 but of infinite length $l \rightarrow \infty$ is also computed. As indicated, the amplitude ratio at low frequencies $\omega \rightarrow 0$ for a non-uniform thermocouple wire of infinite length approaches $\left|T_{1}(0)\right| \rightarrow 1$. This result is identical to the first order frequency response Eq. 13 for a uniform wire of infinite length as is shown in fig. 3 . For the nonuniform wire, however, axial heat transfer does occur at the junction because of dissimilar material properties. At large frequencies $\omega / \omega_{n} \gg 1$, however, the amplitude ratio derived from Eq. (35) for the wire of infinite length asymptotically approaches the value predicted by Eq. (34) for a wire of finite length as shown in fig. 6 . The latter result is similar to the features of fig. 3 at large frequencies for the case of a uniform thermocouple wire. These latter features reflect the fact that the time scales of the ambient gas temperature fluctuations at high frequency are too small for axial heat conduction to occur.

The phase angle $\Phi$ for both expressions Eqs. (34) and (35) is graphed in fig. 7. The phase angle varies over the range $0 \geq \Phi \geq-\pi / 2$ and approaches the lower limit of $-\pi / 2$ for large frequencies. It is apparent that the effect of axial heat conduction is to reduce the magnitude of the phase angle.

The amplitude ratio $\left|T_{1}(0)\right|$ derived from Eq. (34) for a type B thermocouple 
wire is computed in fig. 8. Since the material properties in this case are nearly equal on both sides of the junction, the indicated difference in the amplitude ratio is the result of different wire diameters across the junction. These results are strongly influenced by the magnitude of the factor $Q_{0}$ defined by Eq. (37). If the parameter $Q_{0} \sim 1$ as is the case for the upper curve in fig. 8, both regions 1 and 2 in the schematic of fig. 2 are equally weighted in the expression for the frequency response given by Eq. (34). However, increasing the wire diameter $D_{2}$ in region 2 means $Q_{0}$ is reduced in magnitude such that $Q_{0}<1$. Now the amplitude ratio is more strongly influenced by the properties of the larger wire in region 2 . The phase angle is plotted in fig. 9 for the same wire configuration.

The amplitude ratio $\left|T_{1}(x)\right|$ is also computed as a function of the axial distance down the type $B$ thermocouple wire in fig. 10. This was accomplished by substituting the expression for the constant $A_{1}$ given by Eq. (32) into the general solution for the frequency response Eq. (22). As discussed above, if the ratio $Q_{0}<1$ because of an increase in $D_{2}$, a large increase in the rate of heat transfer occurs in region 2 down the larger wire. This additional axial heat conduction reduces the amplitude ratio at the thermocouple junction compared to the case of $Q_{0} \sim 1$ for the wire geometry represented by the upper curve in fig. 10.

The amplitude ratio derived from Eq. (34) for a type $T$ thermocouple wire is graphed in fig. 11. Since the thermoconductivity of copper in region 1 is more than an order of magnitude larger than the thermoconductivity of constantan in region 2, the parameter $Q_{0}>1$ for $D_{1} / D_{2}=1$. Increasing the diameter of region 2, 
however, has a small effect on the amplitude ratio since $Q_{0}>1$, which remains unchanged, and the properties of region 1 continue to dominate the rate of heat transfer. The phase angle for the same configuration is computed in fig. 12.

The amplitude ratio $\left|T_{1}(x)\right|$ is also computed as a function of the axial distance down the type $T$ thermocouple wire in fig. 13. As discussed above, $Q_{0}>$ 1 for both wire configurations. Thus, the large rate of axial heat transfer down the copper wire in region 1 gives nearly equal spacial temperature profiles for both wire configurations and this fixes the junction temperature. 


\section{NOMENCLATURE}
$A=$ constant of integration
$B=$ constant of integration
c $=$ material specific heat $\left(J-g m^{-1}-{ }^{0} K^{-1}\right)$
$c_{j}=$ term in infinite series $S_{1}, S_{2}$
$d_{j}=$ term in infinite series $S_{1}, S_{2}$
$D=$ thermocouple wire diameter $(\mathrm{cm})$
$G=1+i\left(\omega / \omega_{n}\right)$
$\mathrm{h}=$ heat transfer coefficient $\left(J-c m^{-2}-s^{-1}-{ }^{0} K^{-1}\right)$
$i=$ unit imaginary number $(=\sqrt{-1})$
$\mathrm{k}=$ material thermoconductivity $\left(J-c m^{-1}-s^{-1}-{ }^{0} K^{-1}\right)$
$\mathrm{k}_{\mathrm{f}}=$ gas thermoconductivity $\left(J-c m^{-1}-s^{-1}-{ }^{0} K^{-1}\right)$
$l=$ length of thermocouple wire $(\mathrm{cm})$
$\mathrm{m}_{12}=\left(Q_{0}-1\right) /\left(Q_{0}+1\right)$
$\mathrm{Nu}=\quad$ Nusselt number $\left(=h D / k_{f}\right)$
$\mathrm{Q}=\left(k_{1} D_{1}^{2} q_{1}\right) /\left(k_{2} D_{2}^{2} q_{2}\right)$
$Q_{0}=\left(k_{1} / k_{2}\right)^{.5}\left(D_{1} / D_{2}\right)^{1.25}$
$Q_{-}=\left(k_{1} / k_{2}\right)^{-5}\left(D_{1} / D_{2}\right)^{2}$
$\mathrm{q}=(G / \gamma)^{3}$
$\operatorname{Re}=$ Reynolds number $\left(=\mathrm{vD} / v_{\mathrm{f}}\right)$
$S_{1}=$ infinite sum 


$$
\begin{aligned}
& S_{2}=\text { infinite sum } \\
& t \quad=\text { time (s) } \\
& \mathrm{T}=\text { frequency response } \\
& \mathrm{T}_{\mathrm{f}}=\text { amplitude of periodic gas temperature }\left({ }^{0} \mathrm{~K}\right) \\
& \mathrm{T}_{\mathrm{g}}=\text { gas temperature }\left({ }^{\circ} K\right) \\
& \mathrm{T}_{0}=\text { steady-state gas temperature }\left({ }^{\circ} \mathrm{K}\right) \\
& \vec{T}_{\omega}=\text { complex amplitude of periodic wire temperature }\left({ }^{\circ} K\right) \\
& T_{w}=\quad \text { local wire temperature }\left({ }^{0} K\right) \\
& \mathbf{v}=\text { gas velocity }\left(c m-s^{-1}\right) \\
& x=\text { axial distance from center of wire }(\mathrm{cm}) \\
& \text { Greek Symbols } \\
& \alpha=\text { thermal diffusivity }\left(\mathrm{cm}^{2}-\mathrm{s}^{-1}\right) \\
& \gamma=\alpha / \omega_{n}\left(\mathrm{~cm}^{2}\right) \\
& \gamma^{+}=1 / \sqrt{\gamma_{1}}+1 / \sqrt{\gamma_{2}}\left(\mathrm{~cm}^{-1}\right) \\
& \gamma^{-}=1 / \sqrt{\gamma_{1}}-1 / \sqrt{\gamma_{2}}\left(c m^{-1}\right) \\
& v_{f}=\quad \text { kinematic viscosity of gas }\left(\mathrm{cm}^{2}-\mathrm{s}^{-1}\right) \\
& \omega=\text { angular frequency }\left(s^{-1}\right) \\
& \omega_{n}=\text { natural frequency of wire }(=4 h / \rho c D)\left(s^{-1}\right) \\
& \Phi=\text { phase angle (degrees) } \\
& \psi=\text { normalized temperature at junction } \\
& \rho=\text { material density }\left(\mathrm{gm}-\mathrm{cm}^{-3}\right)
\end{aligned}
$$




\section{REFERENCES}

1. Carslaw, H. S., Introduction to the Mathematical Theory of the Conduction of Heat in Solids, Dover Publications, 2nd edition (1945).

2. Dils, R. R. and Follansbee, P. S., Wide Bandwidth Gas Temperature Measurements in Combustor and Combustor Exhaust Gases, Instrumentation in the Aerospace Industry 21 (B. Washburn, ed.), ISA 76245 (1976).

3. Elmore, D. L., Robinson, W. W. and Watkins, W. B., Dynamic Gas

Temperature Measurement System: Final Report, NASA CR-168267 (1983).

4. Elmore, D.L., Robinson, W. W. and Watkins, W. B., Further Development of the Dynamic Gas Temperature Measurement System: Vol. I - Technical Efforts, NASA CR-179513 (1986).

5. Forney, L. J., Frequency Response of a Thermocouple Wire: Effects of Axial Conduction, NASA / ASEE Case-Lewis Summer Faculty Fellowship Program, 1988 Final Report, pp. 78-79.

6. Fralick, G. C., Correlation of Velocity and Velocity-Density Turbulence in the Exhaust of an Atmospheric Burner, Turbine Engine Hot Section Technology - 1985, NASA CP-2465, pp. 81-85.

7. Fralick, G. C. and Forney, L. J., Frequency Response of a Supported Thermocouple Wire: Effects of Axial Heat Conduction (in preparation, 1990).

8. Hildebrand, F. B., Advanced Calculus for Applications, (2nd Ed), Prentice Hall (1976).

9. Scadron, M.D. and Warshawsky, I., Experimental Determination of Time Constants and Nusselt Numbers for Bare-Wire Thermocouples in HighVelocity Air Streams and Analytic Approximation of Conduction and Radiation Errors, NACA TN-2599 (1952).

10. Touloukian, Powell, Ho and Clemens, (ed.), Thermal Physical Properties of Matter Purdue Research Foundation, Plenum Pub. (1970). 
VI. TABLES AND FIGURES

Table 1 - Uniform Wire Conditions

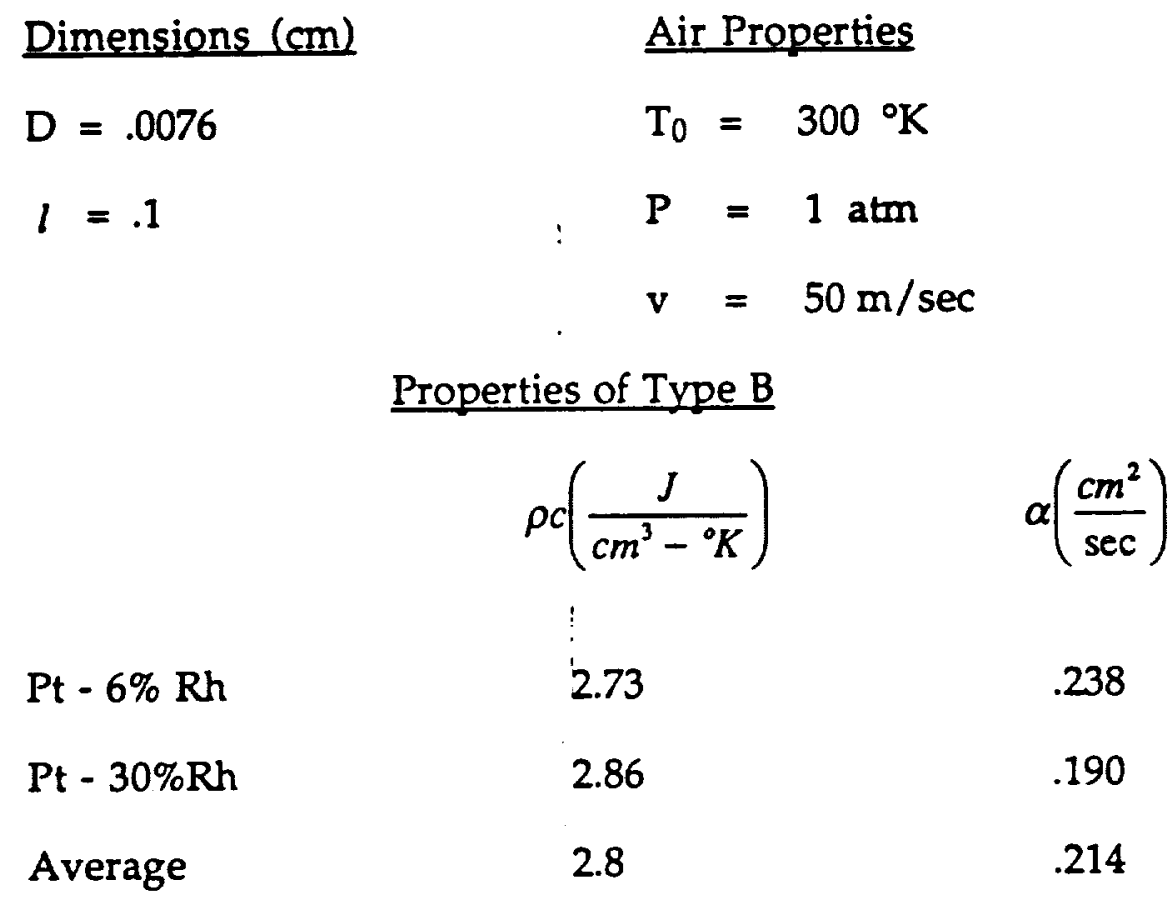

Table 2 - Properties of Non-Uniform Wire

\begin{tabular}{lllllll} 
& \multicolumn{3}{c}{ Dimensions $(\mathrm{cm})$} & & \multicolumn{3}{c}{ Wire Location } \\
$\underline{\mathrm{D}}_{1}(\mathrm{~cm})$ & $\mathrm{D}_{2}(\mathrm{~cm})$ & $\underline{\mathrm{D}}_{2} / \mathrm{D}_{1}$ & $l(\mathrm{~cm})$ & $\underline{\text { Region }}$ & Type B & Type T \\
.0076 & .0076 & 1 & .1 & 1 & Pt-6\%Rh & Copper \\
.0076 & .0152 & 2 & .1 & .2 & Pt-30\%Rh & Constantan
\end{tabular}

Properties of Type T

$$
\rho c\left(\frac{J}{c m^{3}-\cdot K}\right) \quad \alpha\left[\frac{\mathrm{cm}^{2}}{\mathrm{sec}}\right]
$$

Copper

Constantan
3.44

3.48
1.16

.067 


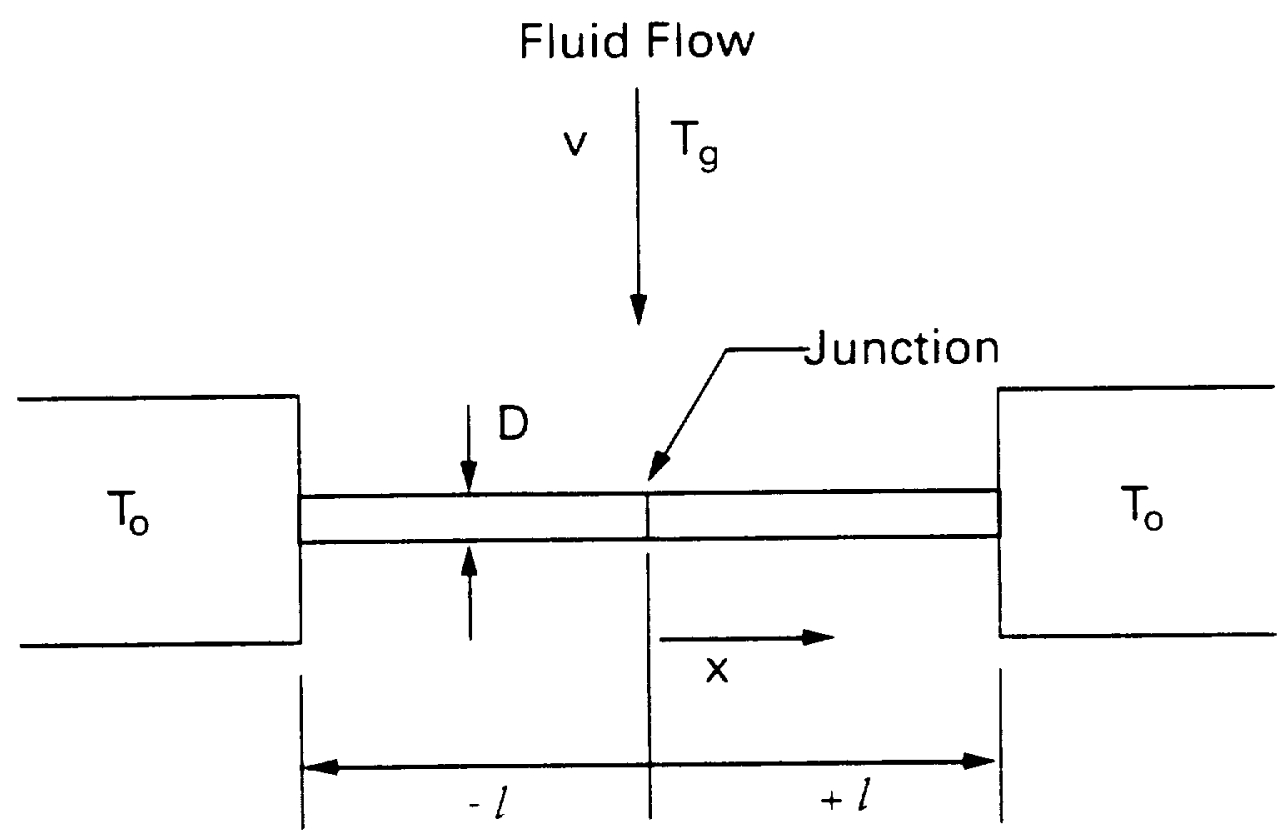

Fig. 1 Schematic of uniform wire

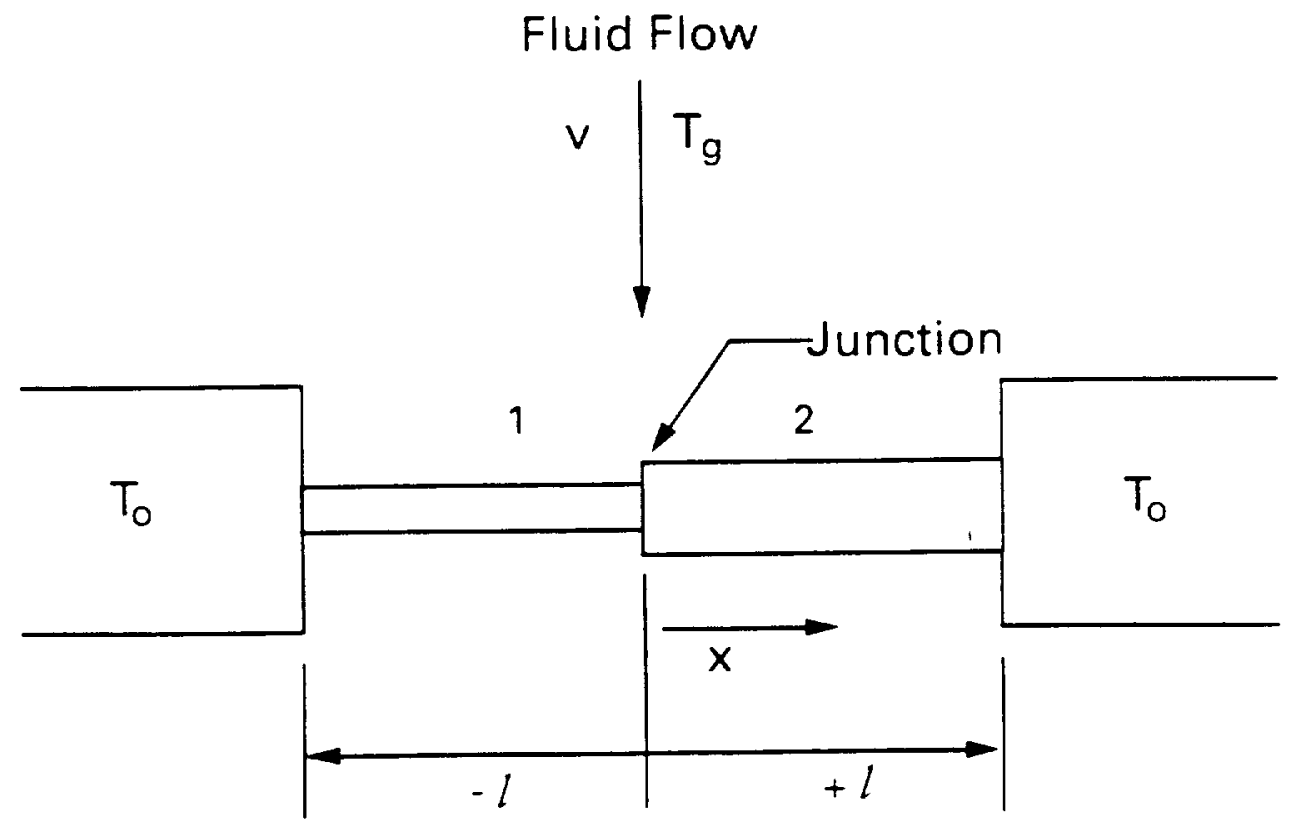

Fig. 2 Schematic of non-uniform wire 


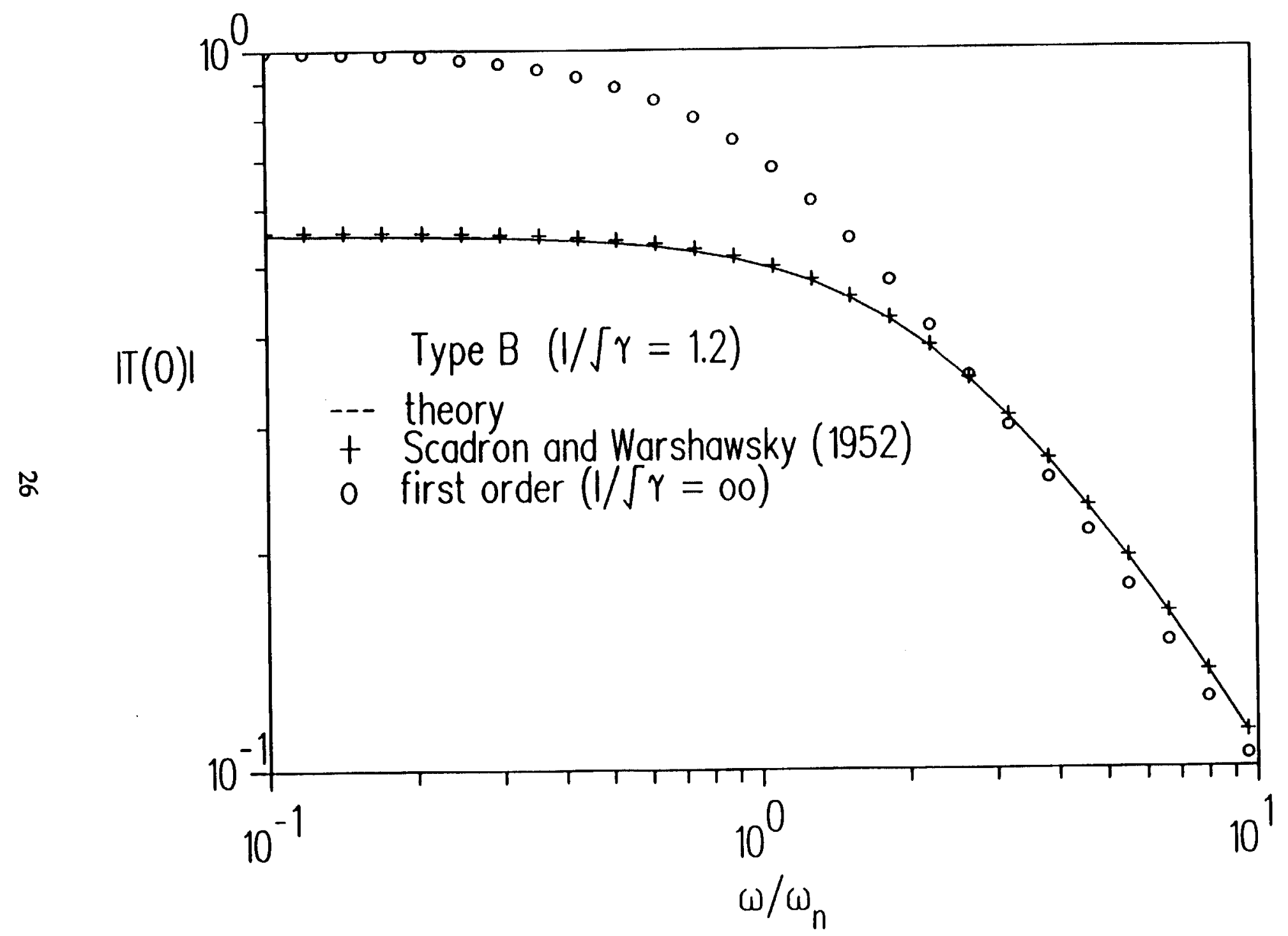

Fig. 3 Amplitude ratio vs angular frequency for uniform wire. Solid line, Eq. (12); Circles, Eq. (13). 


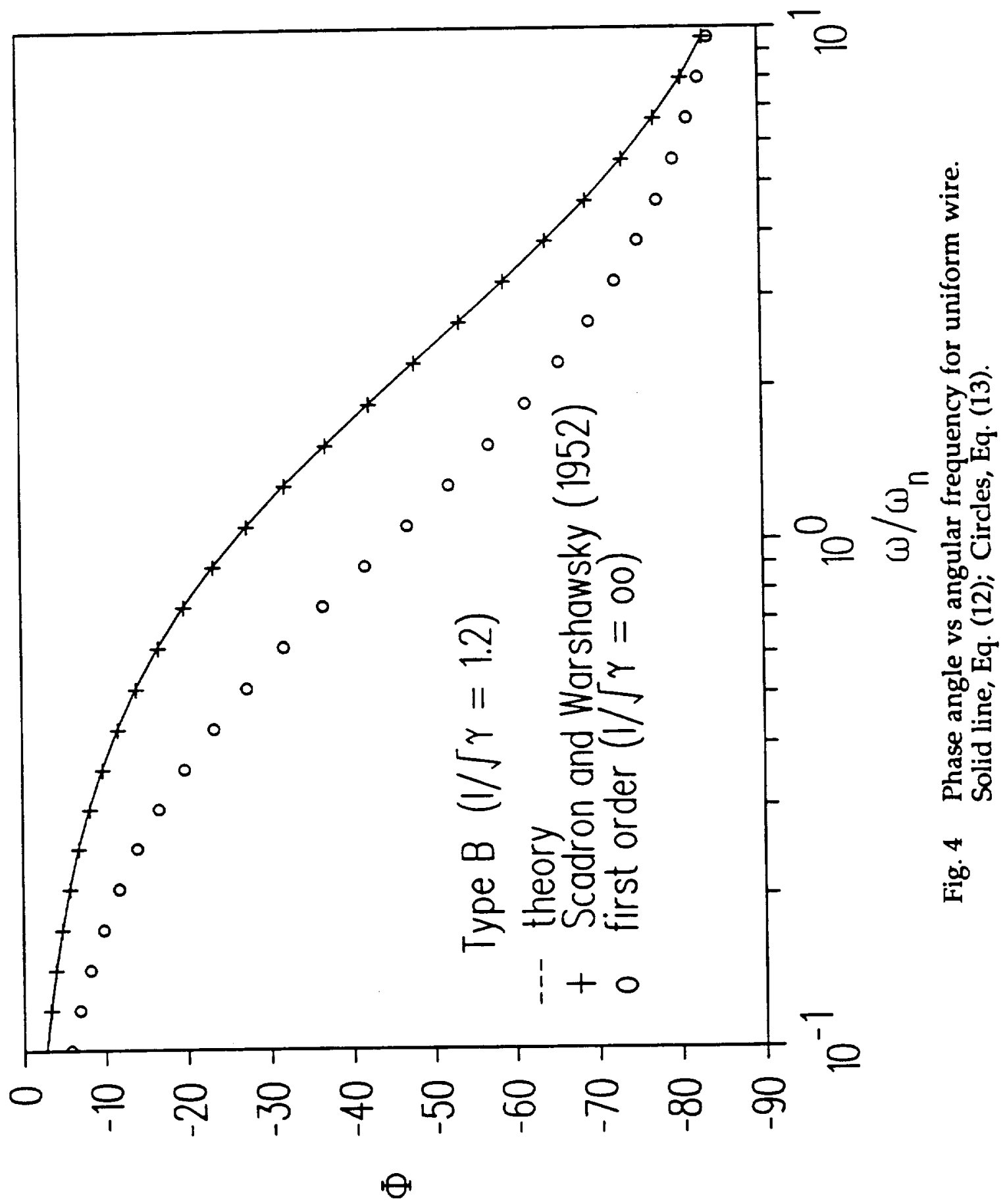




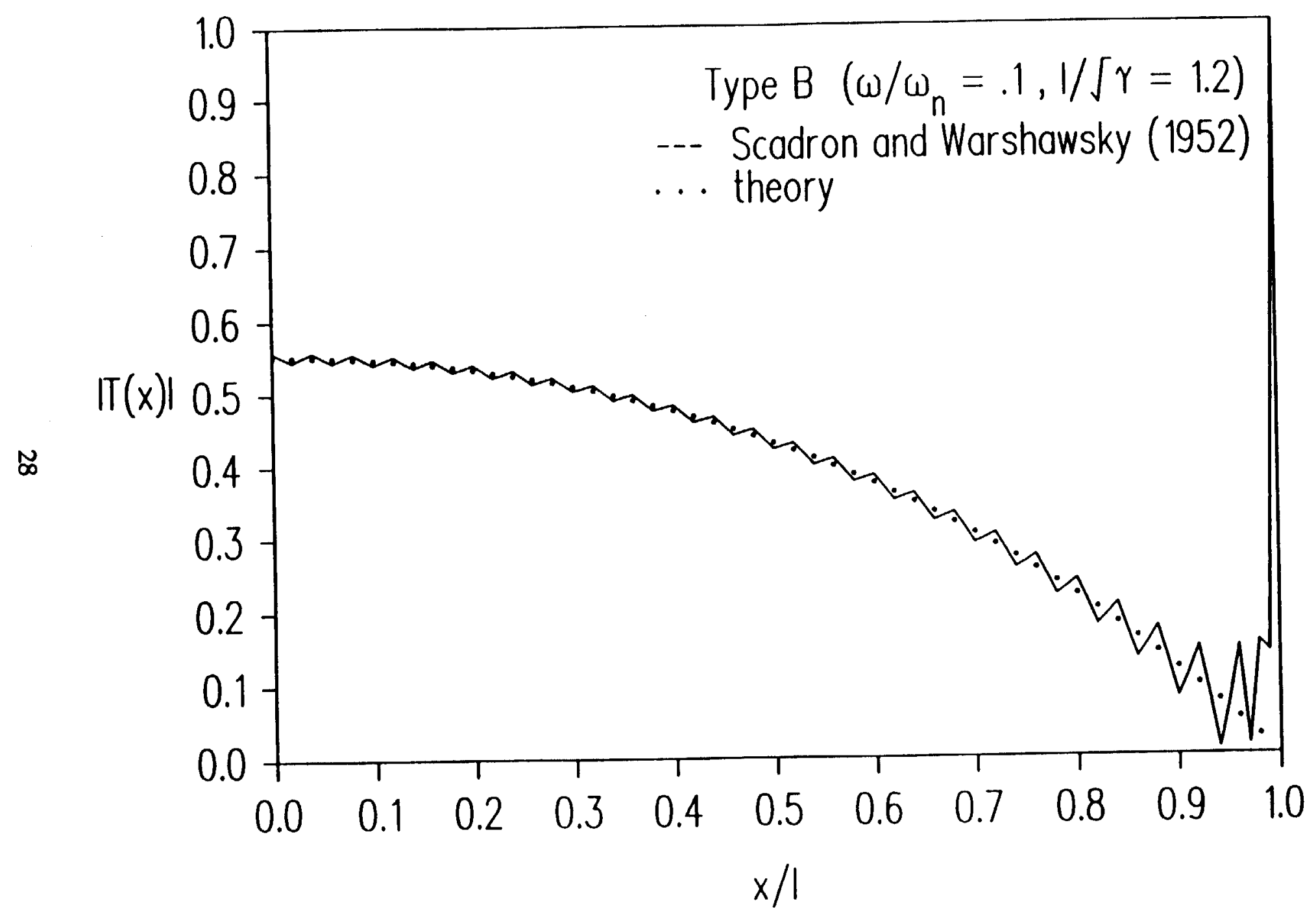

Fig. 5 Amplitude ratio vs axial distance for uniform wire. Dotted line, Eq. (11). 


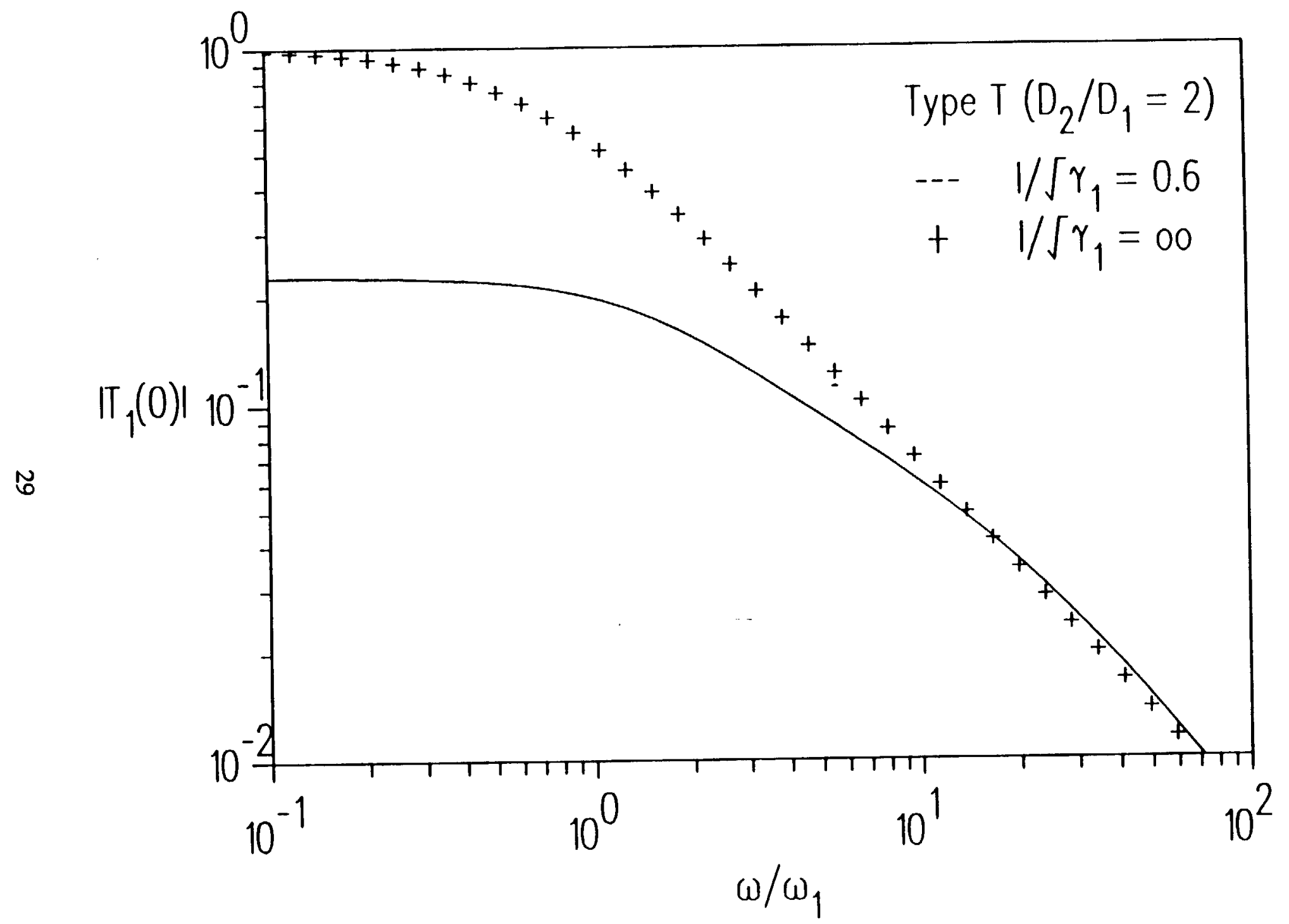

Fig. 6 Amplitude ratio vs angular frequency for non-uniform wire. Upper curve is Eq. (35). Lower curve is Eq. (34). 


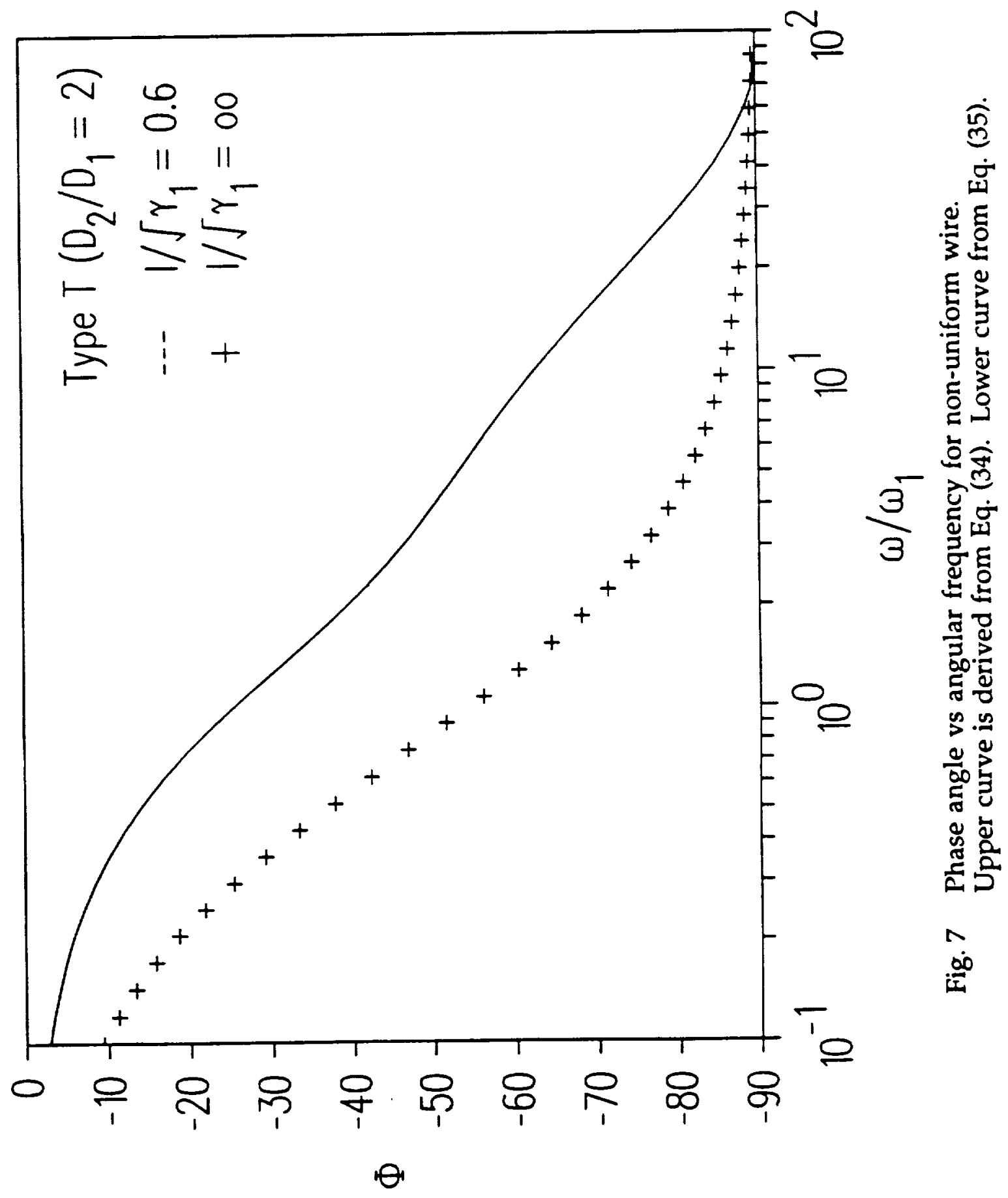




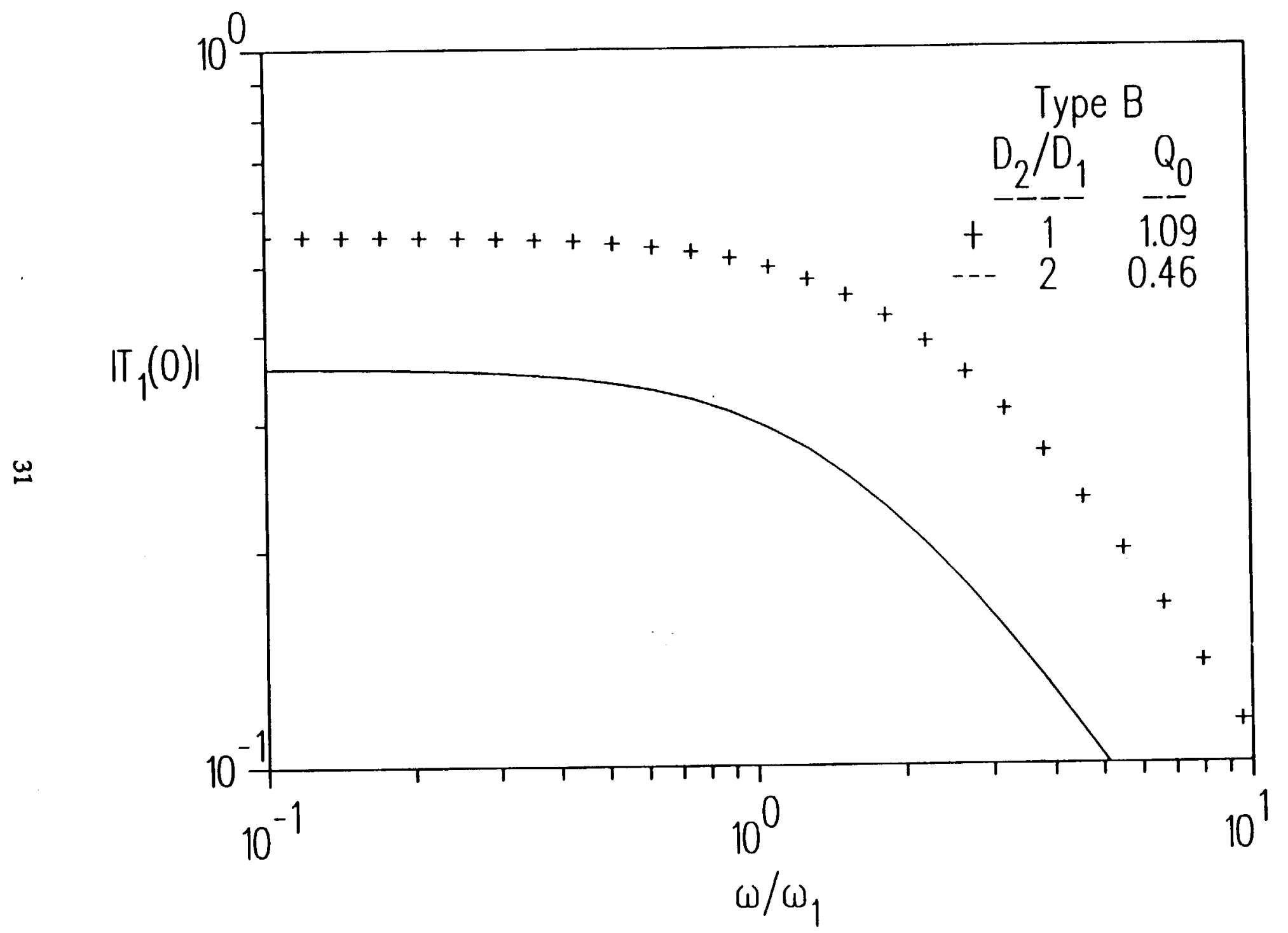

Fig. 8 Amplitude ratio vs angular frequency for non-uniform wire with similar material properties. Curves are Eq. (34). 


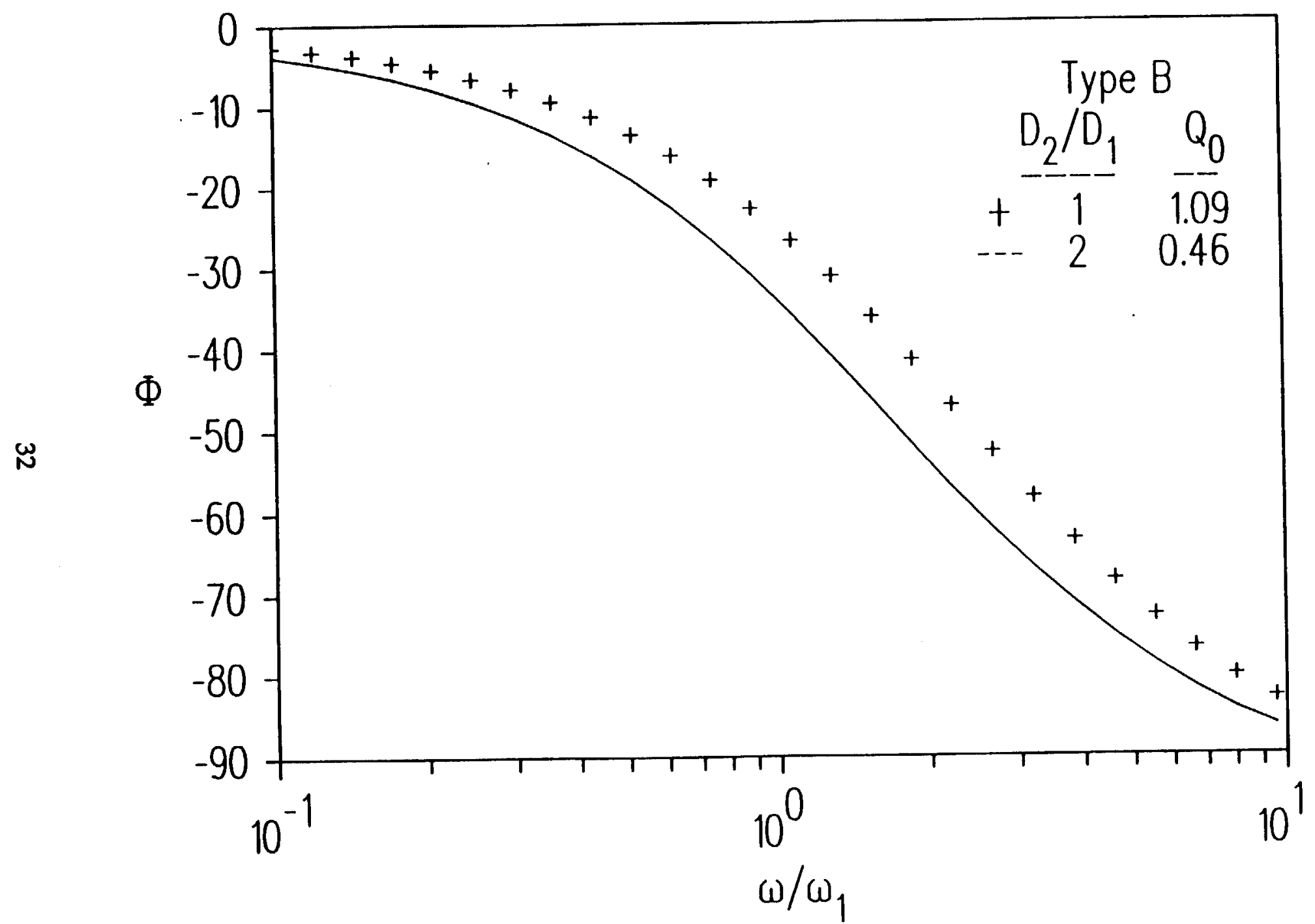

Fig. 9 Phase angle vs angular frequency for non-uniform wire with similar material properties. Curves derived from Eq. (34). 


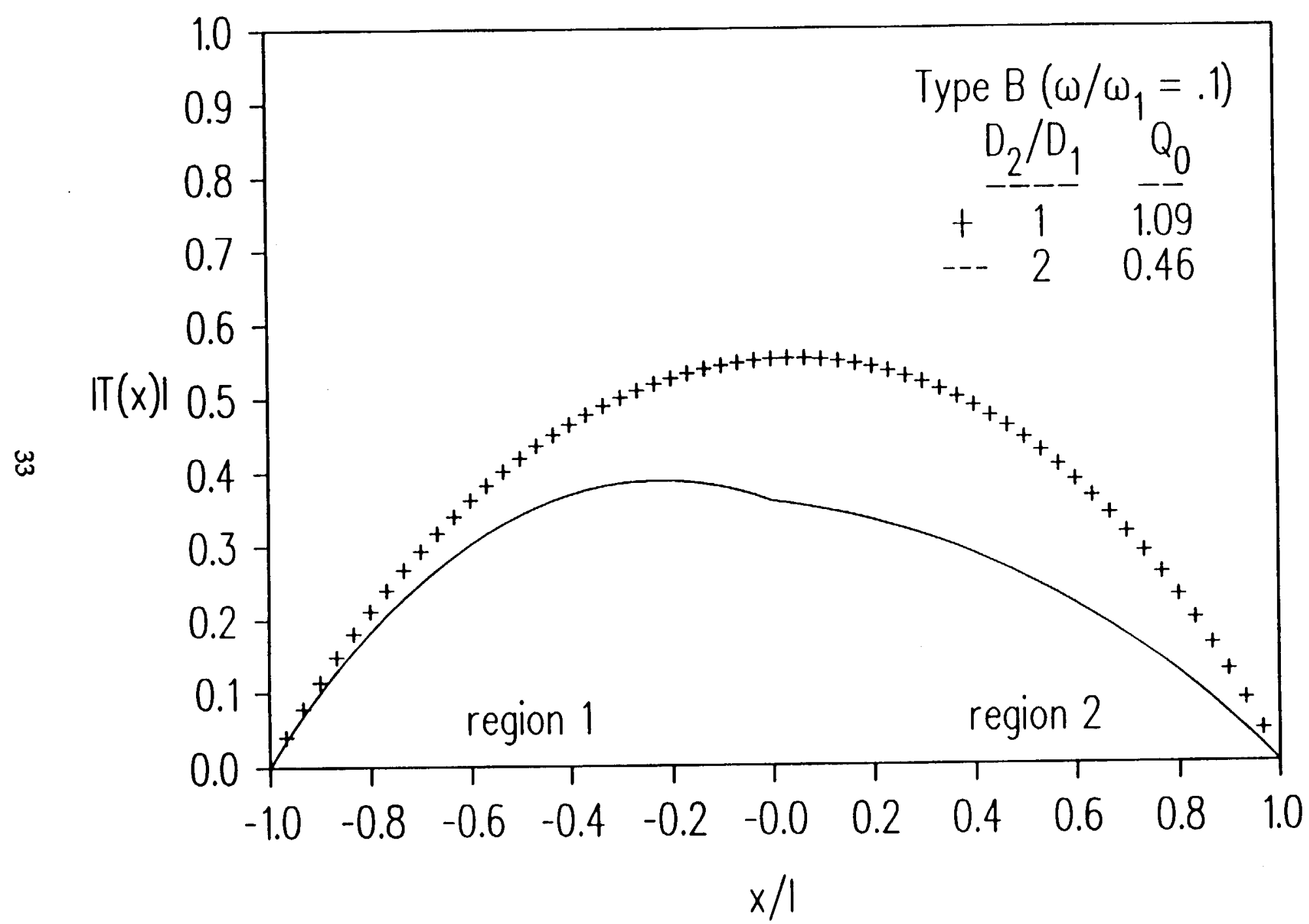

Fig. 10 Amplitude ratio vs axial distance for non-uniform wire with similar material properties. Each curve is derived from Eqs. (22) and (32). 


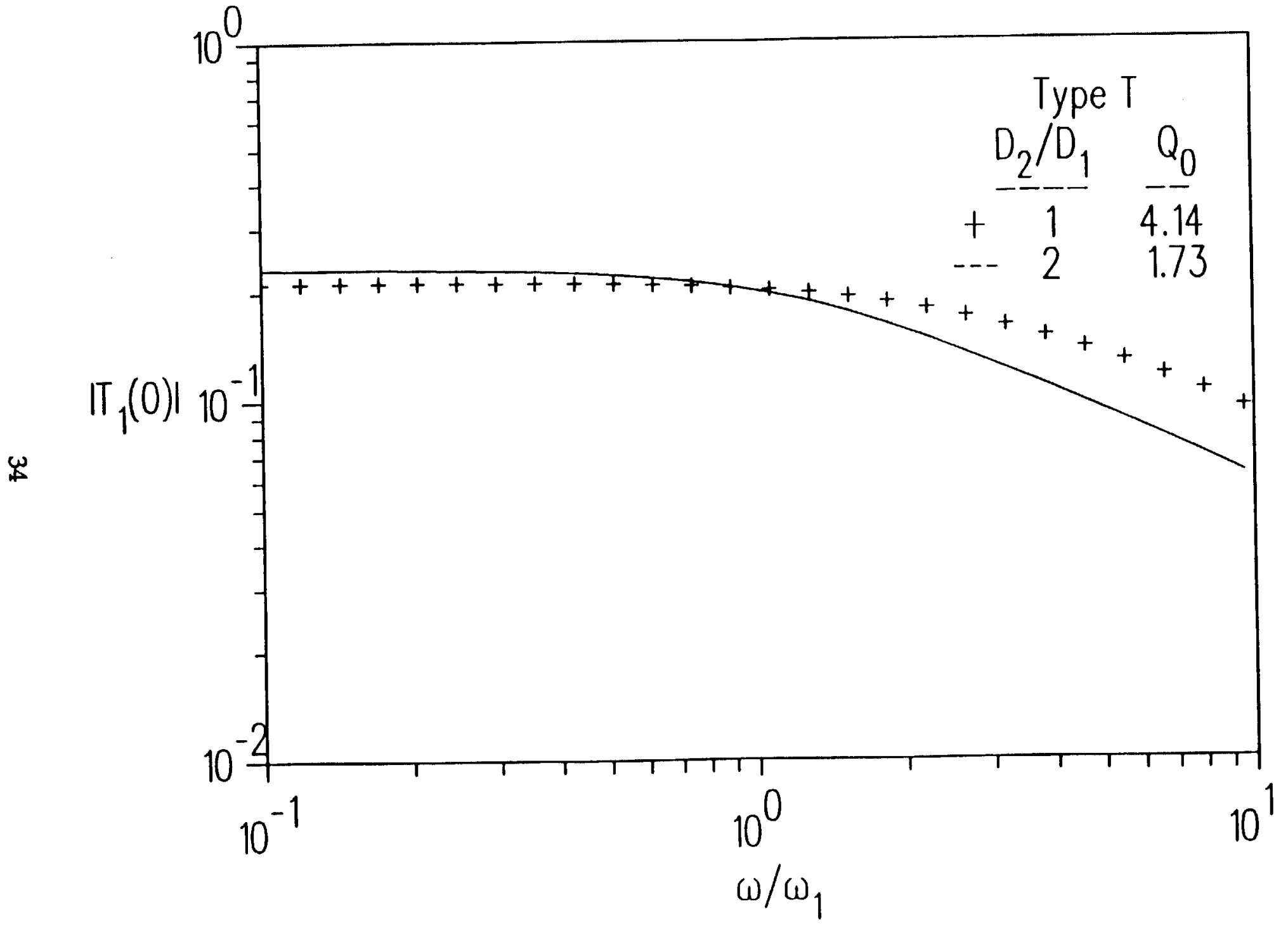

Fig. 11 Amplitude ratio vs angular frequency for wire with dissimilar material properties. Curves are Eq. (34). 


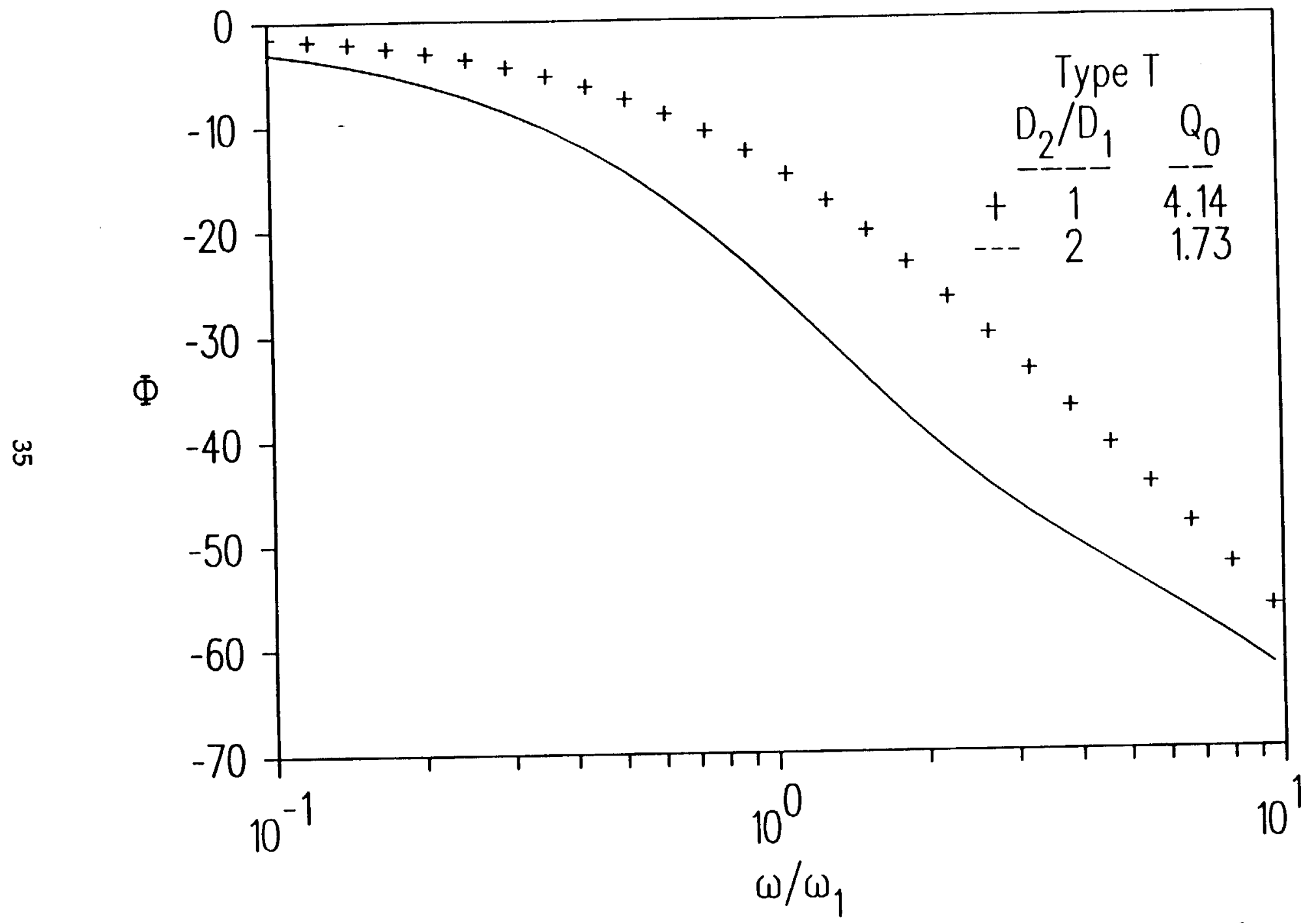

Fig. 12 Phase angle vs angular frequency for wire with dissimilar material properties. Curves derived from Eq. (34). 


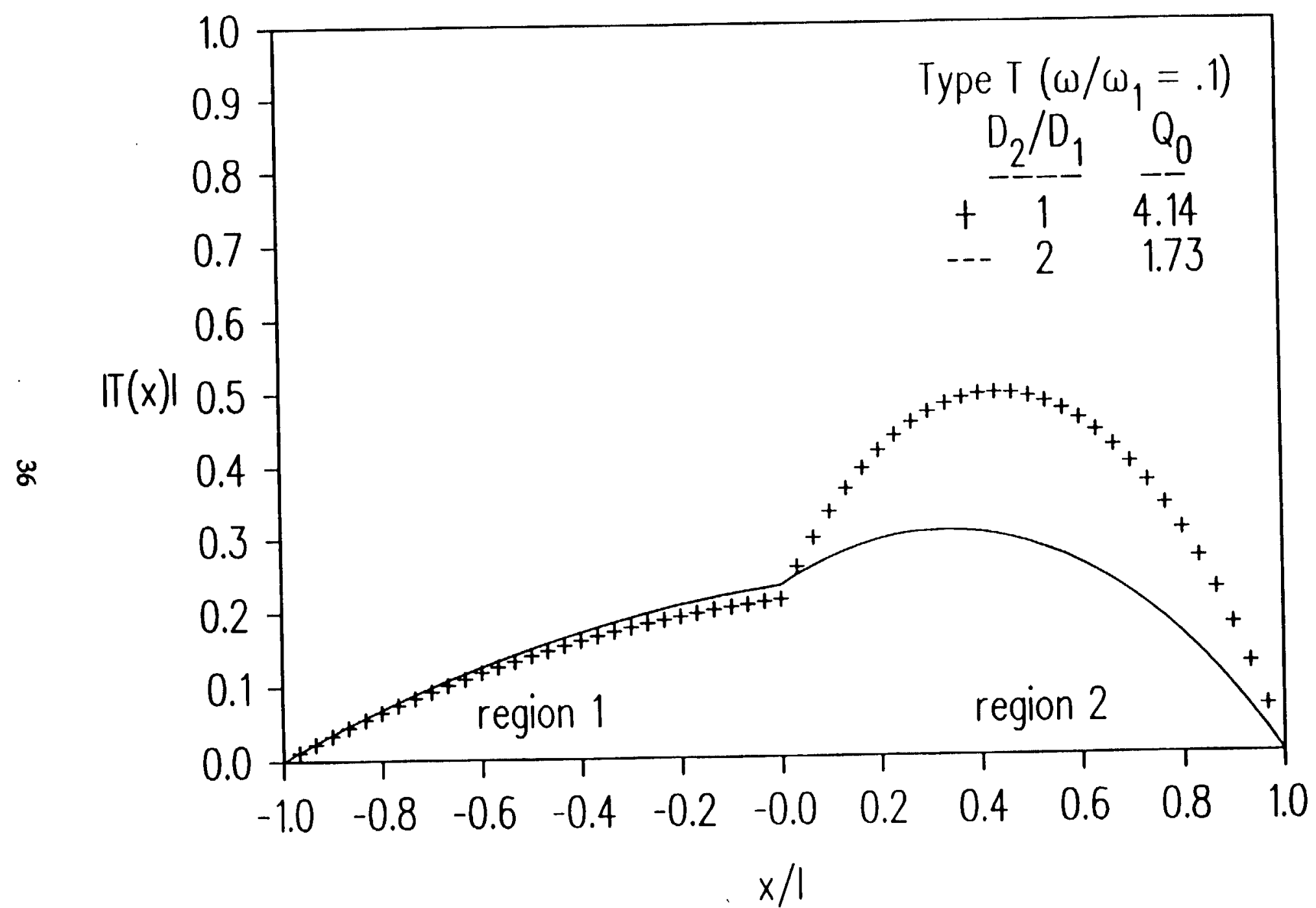

Fig. 13 Amplitude ratio vs axial distance for wire with dissimilar material properties. Each curve is derived from Eqs. (22) and (32). 


\section{FUTURE WORK}

The experimental apparatus to test various thermocouple configurations is fully constructed. Dr. Earl Meeks of the Georgia Tech Microelectronics Laboratory has been given a subcontract to construct the thermocouple probes. During the next 6 months, several thermocouples will be constructed and tested. These thermocuples will consist of either type $\mathrm{K}$ (chromel-alumel) or type $\mathrm{T}$ (copper-constantan) junctions and will have diameter ratios of either 2 or 5 . Among these thermocouples will be several with support legs and some without. These data consisting of amplitude ratio and phase angle will be correlated with various theoretical expressions of the type presented in the present report. 
